\title{
Influence of chronic kidney disease and haemodialysis on stroke outcome
}

Shrikant $D \underline{\text { Pande }^{1}}$, FRCP, FAMS, Julie $\underline{\text { Morris }}^{2}$, MSc

INTRODUCTION Stroke patients with underlying chronic kidney disease (CKD) and those on haemodialysis have complex rehabilitation needs, and their survival and functional outcomes are limited. This study aimed to review post-stroke survival and functional outcomes following rehabilitation in patients with CKD and those on haemodialysis.

METHODS We conducted a retrospective analysis of consecutive stroke patients with underlying CKD (Stages G3b, G4 and $G 5 ; n=30)$ and those on dialysis at the time of stroke $(n=7)$, who underwent in-patient rehabilitation between June 2008 and May 2017. The mean duration of follow-up was 56 months. Demographic details, associated comorbidities and laboratory parameters were reviewed. Baseline and follow-up scores of the National Institute of Health Stroke Scale (NIHSS) and Functional Independence Measure (FIM), and dates of death of the patients were analysed.

RESULTS Of the 37 consecutive stroke patients (mean age 64.7 years), 34 had ischaemic stroke and three had haemorrhagic transformation. Significant improvements in NIHSS and FIM scores were observed from the time of admission to after discharge. Older age, longer duration of hospital stay, lower estimated glomerular filtration rate and low haemoglobin levels were all significantly related to mortality.

CONCLUSION Despite significant functional and neurological improvements following rehabilitation, stroke patients with underlying CKD had higher average duration of hospital stay, more recurrent hospitalisations and poorer survival outcomes than those without underlying CKD. This could be attributed to the complications associated with CKD rather than stroke. Multidisciplinary community rehabilitation may be an alternative to reduce recurrent hospitalisations and morbidity in this group of patients.

Keywords: chronic kidney disease, FIM, haemodialysis, NIHSS, stroke

\section{INTRODUCTION}

Stroke remains a major contributor to inpatient rehabilitation, as it results in significant disabilities and long-term complications. The majority of patients with stroke require long-term follow-up for comorbidities and complications. Studies on survival have focused on various subgroups of stroke. Haemodialysis (HD) is associated with common comorbidities such as diabetes mellitus and hypertension. Patients undergoing dialysis are, thus, susceptible to vascular complications, including stroke. ${ }^{(1)}$ The steady increase in the number of individuals undergoing dialysis is accompanied by an increased risk of stroke.

Both the kidney and the brain require continuous and stable high blood flow in a low vascular resistance system. These two organs are supplied by strain arterioles. Owing to the branching nature of these arterioles, they are susceptible to blood pressure changes. ${ }^{(2,3)}$ The arterial endothelium and tunica media are adversely affected in patients with chronic kidney disease (CKD), making them prone to cerebrovascular insults. ${ }^{(3,4)}$ Further, in patients on HD, the baroreceptor reflex is altered owing to a deranged autonomic function, ${ }^{(5)}$ which results in poor tolerance to fluid and blood pressure changes during dialysis. ${ }^{(6)} \mathrm{HD}$ can also result in myocardial stunning, leading to inadequate cerebral perfusion, which may subsequently lead to ischaemic brain injury. ${ }^{(7)}$ A previously published study from the United Kingdom has demonstrated that patients with haemodynamic instability during dialysis show increased white matter changes (indicative of ischaemic damage). ${ }^{(8)}$ White matter hyperintensities and grey matter loss are also associated with end-stage renal failure (ESRF). ${ }^{(9,10)}$

Patients with CKD are known to have cognitive decline, information processing problems and executive dysfunction. ${ }^{(11-13)}$ Although the presence of CKD is a known risk factor for stroke, ${ }^{(14,15)}$ proteinuria $^{(14)}$ and low glomerular filtration rate (GFR) have also been proven to be independent risk factors for reduced cerebral perfusion and stroke. ${ }^{(16,17)}$ Increased risk of stroke in patients with CKD is a result of uraemia-associated calcification in blood vessels, along with 'malnutrition-inflammation atherosclerosis syndrome' ${ }^{(18}$

In this study, we aimed to identify the rehabilitation outcomes, in terms of gains in the Functional Independence Measure $(\mathrm{FIM})^{(19)}$ and changes in National Institute of Health Stroke Scale $(\mathrm{NIHSS})^{(20)}$ scores, in stroke patients with underlying CKD and those on dialysis. We also analysed the number of recurrent hospitalisations and the duration of hospital stay in relation to survival outcomes.

\section{METHODS}

This was a retrospective study involving consecutive stroke patients who were admitted to the neuro-rehabilitation facility at Changi General Hospital from June 2008 to May 2017. Only patients who met the inclusion criteria were included. All included patients were discharged from the rehabilitation facility and followed up regularly as outpatients for a minimum of three

${ }^{1}$ Department of Rehabilitation Medicine, Changi General Hospital, Singapore, ${ }^{2}$ Manchester University, NHS Foundation Trust, UK

Correspondence: Dr Shrikant D Pande, Consultant, Department of Rehabilitation Medicine, Changi General Hospital, 2 Simei Street 3 , Singapore 529889. shrikant.digambarrao.pande@singhealth.com.sg 
months. The subsequent records of hospital admissions, follow-up changes in general physical and neurological status, and treatment regimens were analysed. The SingHealth Centralised Institutional Review Board approved this study. The requirement of obtaining informed consent from patients was waived owing to the retrospective nature of the study.

Patients with CKD Stages G3b, G4 or G5 (based on the Kidney Disease: Improving Global Outcomes [KDIGO] staging) ${ }^{(21)}$ and those on haemodialysis or peritoneal dialysis when stroke was diagnosed were included. Patients with previous known strokes; incomplete follow-up records; follow-up period of less than three months; and those with normal renal function, acute kidney injury, or CKD Stage G1, G2 or G3a were excluded.

Stroke and its subtypes were diagnosed by a stroke physician or neurosurgeon on admission, based on clinical examination, brain imaging (computed tomography $[\mathrm{CT}]$, magnetic resonance [MR] imaging, magnetic resonance angiography), 12-lead electrocardiography, continuous or Holter monitor, carotid Doppler test and echocardiography.

The medical records of the included patients were reviewed. Data collected included demographic details, diagnosis, type of stroke (e.g. ischaemic, intracerebral bleed), CT/MR imaging findings for stroke territory, admission electrolytes, clotting profiles, premorbid medications and comorbidities. Treatment modalities included thrombolysis, medical treatments for raised intracranial pressure, neurosurgical interventions and statins.

Categorical data was presented as frequency (percentage), while continuous data was presented as mean $( \pm$ standard deviation) for parametric distributions and median (interquartile range) for non-parametric distributions. Differences in characteristics were examined using the chi-square test for categorical variables and the two-sample $t$-test or Mann-Whitney $U$ test for continuous variables, where appropriate. Logistic regression analysis was performed to determine the association between potential predictors and outcomes of seizure. Odds ratio was presented along with $95 \%$ confidence intervals (Cls). A twotailed p-value $<0.05$ was considered statistically significant. Analysis was performed using IBM SPSS Statistics version 19.0 (IBM Corp, Armonk, NY, USA).

\section{RESULTS}

Of the 563 records of patients with stroke that were reviewed, 37 met the selection criteria. Of these, 30 (81\%) patients had CKD (Stages G3b, G4, G5) and seven (19\%) were on dialysis at the time of stroke. The median duration of follow-up was 56 (range 20-93) months. Table I shows the patients' demographic and clinical characteristics. The mean age of the patients was 64.7 (range $36-87)$ years; $44 \%(n=16)$ of the patients were male and $73 \%(n=27)$ had diabetes mellitus. The mean duration of hospital stay for the initial stroke was 28 (range 4-77) days. Using the Oxfordshire Community Stroke Project classification, ${ }^{(22)}$ the distribution of stroke territory was as follows: partial anterior circulation stroke $(n=23)$, posterior circulation stroke $(n=8)$, total anterior circulation stroke
Table I. Patient demographic and clinical characteristics $(\mathbf{n}=\mathbf{3 7})$.

\begin{tabular}{|ll|}
\hline Characteristic & Mean \pm SD (range)/No. (\%) \\
\hline Age (yr) & $64.7 \pm 13.1(36-87)$ \\
\hline Male gender & $16(44)$ \\
\hline Duration of hospital stay (day) & $28.0(4-77)$ \\
\hline Diabetes mellitus & $27(73)$ \\
\hline Comorbidities* & $5(14)$ \\
\hline Estimated glomerular filtration rate & $21.3 \pm 12.3(5-44)$ \\
\hline Haemoglobin (g/dL) & $11.9 \pm 2.0(9-18)$ \\
\hline Urea (mmol/L) & $12.1 \pm 6.1(3.8-26.0)$ \\
\hline Albumin (g/L) & $29.9 \pm 5.2(15-39)$ \\
\hline
\end{tabular}

${ }^{*}$ Comorbidities include breast cancer, colon cancer, rectal cancer, cirrhosis and gastrointestinal stromal tumour (three out of five patients died during follow-up). SD: standard deviation

Table II. Relationship between variables and survival.

\begin{tabular}{|c|c|c|}
\hline & \multicolumn{2}{|c|}{ Univariate analysis* } \\
\hline & Unadjusted HR (95\% Cl) & p-value \\
\hline Age & $1.05(1.01-1.09)$ & $0.014^{\dagger}$ \\
\hline Male gender & $0.59(0.23-1.54)$ & 0.28 \\
\hline $\begin{array}{l}\text { Duration of } \\
\text { hospital stay }\end{array}$ & $1.03(1.01-1.06)$ & $0.010^{\dagger}$ \\
\hline Diabetes mellitus & $0.55(0.19-1.56)$ & 0.26 \\
\hline eGFR & $0.96(0.92-0.999)$ & $0.046^{\dagger}$ \\
\hline Haemoglobin & $0.75(0.56-1.001)$ & 0.051 \\
\hline Urea & $1.03(0.95-1.12)$ & 0.46 \\
\hline Albumin & $0.93(0.85-1.02)$ & 0.13 \\
\hline
\end{tabular}

*Using Cox proportional hazards regression. $†$ Statistically significant at $\mathrm{p}<0.05$. $\mathrm{Cl}$ : confidence interval; eGFR: estimated glomerular filtration rate; HR: hazard ratio

$(\mathrm{n}=3)$ and haemorrhagic transformation $(\mathrm{n}=3)$. The mean values on admission were: estimated glomerular filtration rate (eGFR) 21.3 (range 5-44); haemoglobin 11.9 (range 9-18) g/ $\mathrm{dL}$, urea 12.1 (range 3.8-26.0) $\mathrm{mmol} / \mathrm{L}$ and albumin 29.9 (range 15-39) g/L.

A significant decrease in the NIHSS score was observed from the time of admission to the time after discharge (6 [range 2-19] vs. 4 [range 1-17]; $p<0.001$; Wilcoxon signed rank test). Significant increases in the FIM motor score (31 [range 13-87] vs. 50.5 [range 13-91], $\mathrm{p}<0.001$; Wilcoxon signed rank test) and FIM cognition score (24.6 [range 5-35] vs. 26.6 [range 5-35], $\mathrm{p}=0.046$; paired $t$-test) were observed from the time of admission to the time of discharge.

The median number of recurrent admissions after discharge from the initial admission for stroke (i.e. initial diagnosis) was 6 (range 1-50), and the median duration of hospital stay was 48 (2-291) days. Older age, longer duration of hospital stay and lower eGFR were all significantly related to mortality $(p<0.05)$, while lower haemoglobin levels showed borderline significance ( $p=0.051$; Table II). These four factors were further assessed using multivariable Cox regression analysis to identify the optimal subset of independent predictors of mortality. Age, duration of hospital stay and haemoglobin levels were found to have significant independent relationships with mortality. 


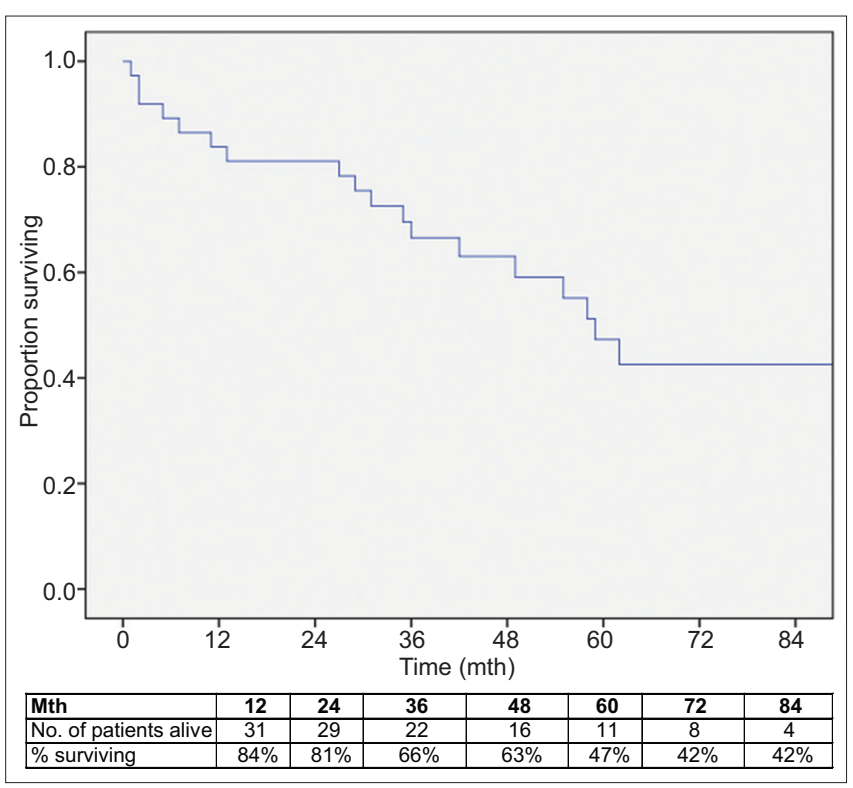

Fig. 1 Graph shows the survival outcome of stroke patients with chronic kidney disease/end-stage renal failure.

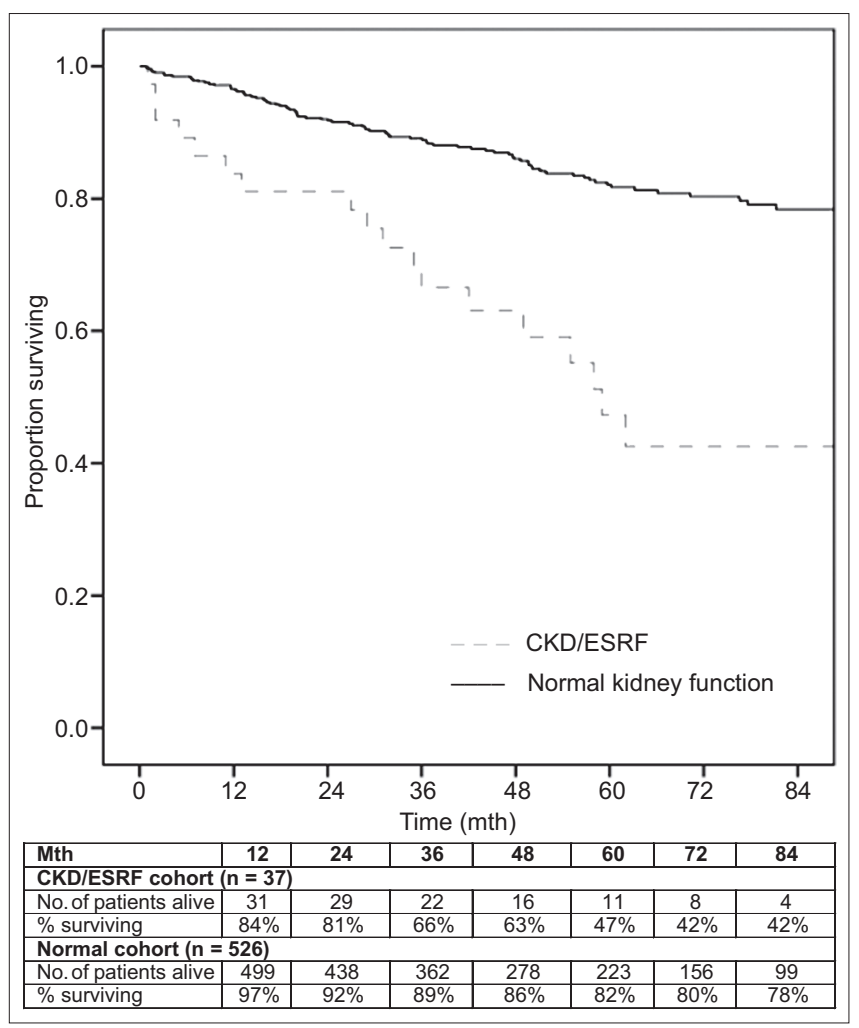

Fig. 2 Graph shows survival outcomes of stroke patients with chronic kidney disease/end-stage renal failure (CKD/ESRF cohort) vs. stroke patients with normal kidney function (normal cohort). The former shows significantly higher mortality (hazard ratio $3.5,95 \%$ confidence interval $2.1-5.8, p<0.001)$

Five patients in the dialysis group and 13 patients in the CKD group died during the follow-up period. Among those who died, three patients had underlying malignancy and one had liver cirrhosis. Significantly higher mortality was observed in the dialysis group (hazard ratio [HR] 3.8, 95\% Cl 1.2-11.4, $\mathrm{p}=0.019$; Fig. 1). Despite adjusting for age, duration of hospital stay and haemoglobin level, higher mortality still persisted in the dialysis group (HR 3.1, 95\% Cl 0.9-11.5, p = 0.08), although this was not statistically significant.

The duration of hospital stay did not significantly differ between patients in the dialysis group and those in the CKD group ( $p=0.28$; Mann-Whitney $U$ test). The change in NIHSS score between the time of admission and discharge also did not significantly differ between the two groups ( $p=0.31$; MannWhitney $U$ test). The FIM motor score on admission was of borderline significance between patients on dialysis and those with $\mathrm{CKD}$ (score $=31$ vs. 19, $\mathrm{p}=0.06$; Mann-Whitney $U$ test). The change in FIM motor score between the time of admission and discharge also did not significantly differ between the two groups ( $p=0.95$; Mann-Whitney $U$ test). The difference in FIM cognitive score on admission between the two groups was not significant ( $p=0.44$; independent $t$-test), and the change in FIM cognitive score between the time of admission and discharge also did not significantly differ between the two groups $(p=0.76$; independent $t$-test).

\section{DISCUSSION}

Although HD remains the mainstay of treatment in patients with ESRF, ${ }^{(23)}$ Murray et al concluded that the number of stroke events within the first month of HD increased by a factor of $7 .^{(24)}$ This could be attributed to decreased cerebral perfusion and an altered rate of blood flow. ${ }^{(24-26)}$ Other factors that may contribute to an increased risk of stroke at the initiation of HD are advancing age, diabetes mellitus, hypertension, ${ }^{(18)}$ use of erythropoiesisstimulating agent, blood viscosity and peripheral vascular resistance. ${ }^{(24)}$ In the present study, the number of patients with stroke on HD was small $(n=7)$ compared to those with CKD $(\mathrm{n}=30)$.

Compared to the general population, patients on HD have about ten times ${ }^{(23)}$ increased risk of stroke (with 20\%-30\% being haemorrhagic strokes) ${ }^{(27,28)}$ and an increased risk of haemorrhagic transformation, with more haematoma volume in haemorrhagic stroke. ${ }^{(29)}$ In the present study, 34 patients had ischaemic strokes, two patients received recombinant tissue plasminogen activator and three were found to have haemorrhagic transformation. Seven patients in our study had underlying atrial fibrillation and 12 had recurrence of stroke during the follow-up period. In contrast to research conducted elsewhere, studies conducted in Japan have demonstrated a high incidence of haemorrhagic stroke, which can be explained by platelet dysfunction, heparin use and the fact that the Japanese population has a higher risk of haemorrhagic stroke compared to other populations. ${ }^{(30,31)}$ Thrombolysis for the treatment of stroke in patients with ESRF and CKD has shown conflicting results, with some studies suggesting an increased risk of haemorrhage ${ }^{(32-34)}$ and others reporting increased mortality without increased bleeding risk. ${ }^{(35)}$ In our study, however, none of the patients had intracerebral haemorrhage.

Ovbiagele et al, in a series of 94 patients with recent intracerebral haemorrhage, found that patients who were discharged home were less likely to have proteinuria or low eGFR; 
however, the authors did not find any statistical significance with regard to these variables. ${ }^{(36)}$ In a large series that examined the rehabilitation outcomes of elderly patients, Doyle et al found that eGFR and the Barthel score were independent risk factors for survival. ${ }^{(37)}$ In the present study, two of our patients received recombinant tissue plasminogen activator, and only one of them had haemorrhagic transformation.

It is observed that patients with CKD and stroke have poorer survival and functional outcomes. When we compared the data of 526 stroke patients without renal dysfunction seen in our hospital with our cohort of stroke patients with underlying $\mathrm{CKD}$ or $\mathrm{HD}$, the latter were found to have longer duration of hospital stay, more recurrent hospitalisations and poorer survival outcomes (Fig. 2).

Previous studies have reviewed cerebral protection during $\mathrm{HD}$. Eldehni et al concluded that dialysis performed at $0.5^{\circ} \mathrm{C}$ below a patient's core temperature was associated with better haemodynamic stability and protection against white matter ischaemic changes. ${ }^{(8)}$ Continuous, instead of intermittent dialysis, has also been suggested to reduce the incidence of brain injury. ${ }^{(1)}$

The present study was performed at a government restructured hospital. The admitted patients represented all socioeconomic levels from a local catchment area. Therefore, differences in socioeconomic status and ethnicity were not likely to have influenced our results, and this is a major strength of the study. However, our study was limited by its retrospective, observational and single-centre design. Further, patients who did not undergo rehabilitation were excluded from our study.

In conclusion, our study found that despite significant improvements in NIHSS and FIM scores, patients with stroke and CKD who undergo HD continue to have poor survival. The average duration of hospital stay and recurrent hospitalisation rate in this group of patients are also higher than those in stroke patients without CKD. Patients with HD tend to have recurrent admissions and higher morbidity owing to fistularelated problems. The morbidity and mortality in patients with stroke and CKD who are on HD may also be attributable to the complications associated with these comorbidities rather than the stroke itself. Multidisciplinary community rehabilitation that focuses on preventing and addressing these issues may help to reduce recurrent hospitalisations in this group of patients.

\section{ACKNOWLEDGEMENTS}

We would like to thank Geraldine Lim, Clinical Trials and Research Unit, Department of Rehabilitation Unit and Rehabilitation Services, Changi General Hospital, for her assistance.

\section{REFERENCES}

1. Davenport A. Practical guidance for dialyzing a hemodialysis patient following acute brain injury. Hemodial Int 2008; 12:307-12.

2. Lau WL, Huisa BN, Fisher M. The cerebrovascular-chronic kidney disease connection: perspectives and mechanisms. Transl Stroke Res 2017; 8:67-76.
3. Naganuma T, Takemoto Y. New aspects of cerebrovascular diseases in dialysis patients. Contrib Nephrol 2015; 185:138-46.

4. Toyoda K, Ninomiya T. Stroke and cerebrovascular diseases in patients with chronic kidney disease. Lancet Neurol 2014; 13:823-33.

5. Chesterton LJ, Sigrist MK, Bennett T, Taal MW, McIntyre CW. Reduced baroreflex sensitivity is associated with increased vascular calcification and arterial stiffness. Nephrol Dial Transplant 2005; 20:1140-7.

6. Chesterton LJ, Selby NM, Burton JO, et al. Categorization of the hemodynamic response to hemodialysis: the importance of baroreflex sensitivity. Hemodial Int 2010; 14:18-28.

7. Burton JO, Jefferies HJ, Selby NM, McIntyre CW. Hemodialysis-induced cardiac injury: determinants and associated outcomes. Clin J Am Soc Nephrol 2009; 4:914-20.

8. Eldehni MT, Odudu A, Mclntyre CW. Randomized clinical trial of dialysate cooling and effects on brain white matter. J Am Soc Nephrol 2015; 26:957-65.

9. Eldehni MT, McIntyre CW. Are there neurological consequences of recurrent intradialytic hypotension? Semin Dial 2012; 25:253-6.

10. Prohovnik I, Post J, Uribarri J, et al. Cerebrovascular effects of hemodialysis in chronic kidney disease. J Cereb Blood Flow Metab 2007; 27:1861-9.

11. Sarnak MJ, Tighiouart H, Scott TM, et al. Frequency of and risk factors for poor cognitive performance in hemodialysis patients. Neurology 2013; 80:471-80.

12. Murray AM, Tupper DE, Knopman DS, et al. Cognitive impairment in hemodialysis patients is common. Neurology 2006; 67:216-23.

13. Weiner DE, Scott TM, Giang LM, et al. Cardiovascular disease and cognitive function in maintenance hemodialysis patients. Am J Kidney Dis 2011; 58:773-81.

14. Lee M, Saver JL, Chang $\mathrm{KH}$, et al. Low glomerular filtration rate and risk of stroke: meta-analysis. BMJ 2010; 341:c4249.

15. Ninomiya T, Perkovic V, Verdon C, et al. Proteinuria and stroke: a meta-analysis of cohort studies. Am J Kidney Dis 2009; 53:417-25.

16. Go AS, Fang MC, Udaltsova N, et al; ATRIA Study Investigators. Impact of proteinuria and glomerular filtration rate on risk of thromboembolism in atrial fibrillation: the anticoagulation and risk factors in atrial fibrillation (ATRIA) study. Circulation 2009; 119:1363-9.

17. Sedaghat S, Vernooij MW, Loehrer E, et al. Kidney function and cerebral blood flow: the Rotterdam study. J Am Soc Nephrol 2015; 27:715-21.

18. Power A. Stroke in dialysis and chronic kidney disease. Blood Purif 2013; 36:179-83.

19. Keith RA, Granger CV, Hamilton BB, Sherwin FS. The functional independence measure: a new tool for rehabilitation. Adv Clin Rehabil 1987; 1:6-18.

20. Brott T, Adams H P, Olinger C P, et.al. Measurements of acute cerebral infarction: a clinical examination scale. Stroke 1989; 20:864-870.

21. KDIGO 2012 Clinical Practice Guideline for the Evaluation and Management of Chronic Kidney Disease. Kidney Int Suppl 2013; 3:1-150. Available at: https:// kdigo.org/wp-content/uploads/2017/02/KDIGO_2012_CKD_GL.pdf. Accessed March 29, 2020.

22. Bamford J, Sandercock P, Dennis M, Burn J, Warlow C. Classification and natural history of clinically identifiable subtypes of cerebral infarction. Lancet 1991; 337:1521-6.

23. Belmer L, de Francisco AL, Bueno L, et al. Strokes in patients on haemodialysis: incidence, onset time and associated factors. Nefrologia 2014; 34:347-52.

24. Murray AM, Seiger S, Lakshminarayan K, Herzog CA, Solid CA. Incidence of stroke before and after dialysis initiation in older patients. J Am Soc Nephrol 2013; 24:1166-73.

25. Postiglione A, Faccenda F, Gallotta G, Rubba P, Federico S. Changes in middle cerebral artery blood velocity in uremic patients after hemodialysis. Stroke 1991; 22:1508-11.

26. Hata R, Matsumoto M, Handa N, et al. Effects of hemodialysis on cerebral circulation evaluated by transcranial Doppler ultrasonography. Stroke 1994; 25:408-12.

27. Foley RN, Gilbertson DT, Murray T, Collins AJ. Long intradialytic interval and mortality among patients receiving hemodialysis. N Engl J Med 2011; 365:1099-107.

28. Power A, Chan K, Singh SK, Taube D, Duncan N. Appraising stroke risk in maintenance hemodialysis patients: a large single-center cohort study. Am J Kidney Dis 2012; 59:249-57.

29. Molshatzki N, Orion D, Tsabari R, et al. Chronic kidney disease in patients with acute intracerebral hemorrhage: association with large hematoma volume and poor outcome. Cerebrovasc Dis 2011; 31:271-7.

30. Iseki K, Kinjo K, Kimura Y, Osawa A, Fukiyama K. Evidence for high risk of cerebral hemorrhage in chronic dialysis patients. Kidney Int 1993; 44:1086-90.

31. Iseki K, Fukimaya K. Predictors of stroke in patients receiving chronic hemodialysis. Kidney Int 1996; 50:1672-5.

32. Agrawal V, Rai B, Fellows J, McCullough PA. In-hospital outcomes with thrombolytic therapy in patients with renal dysfunction presenting with acute ischaemic stroke. Nephrol Dial Transplant 2010; 25:1150-7. 
33. Naganuma $M$, Koga $M$, Shiokawa $Y$, et al. Reduced estimated glomerular filtration rate is associated with stroke outcome after intravenous rt-PA: the Stroke Acute Management with Urgent Risk-Factor Assessment and Improvement (SAMURAI) rt-PA registry. Cerebrovasc Dis 2011; 31:123-9.

34. Power A, Epstein D, Cohen D, et al. Renal impairment reduced the efficacy of thrombolytic therapy in acute ischemic stroke. Cerebrovasc Dis 2013; 35:45-52.

35. Tariq N, Adil MM, Saeed F, Chaudhry SA, Qureshi Al. Outcomes of thrombolytic treatment for acute ischemic stroke in dialysis-dependent patients in the United States. J Stroke Cerebrovasc Dis 2013; 22:e354-9.

36. Ovbiagele B, Pineda S, Saver JL. Renal dysfunction and discharge destination in patients with intracerebral hemorrhage. J Stroke Cerebrovasc Dis 2011; 20:145-9.

37. Doyle EM, Sloan JM, Goodbrand JA, et al. Association between kidney function, rehabilitation outcome, and survival in older patients discharged from inpatient rehabilitation. Am J Kidney Dis 2015; 66:768-74.

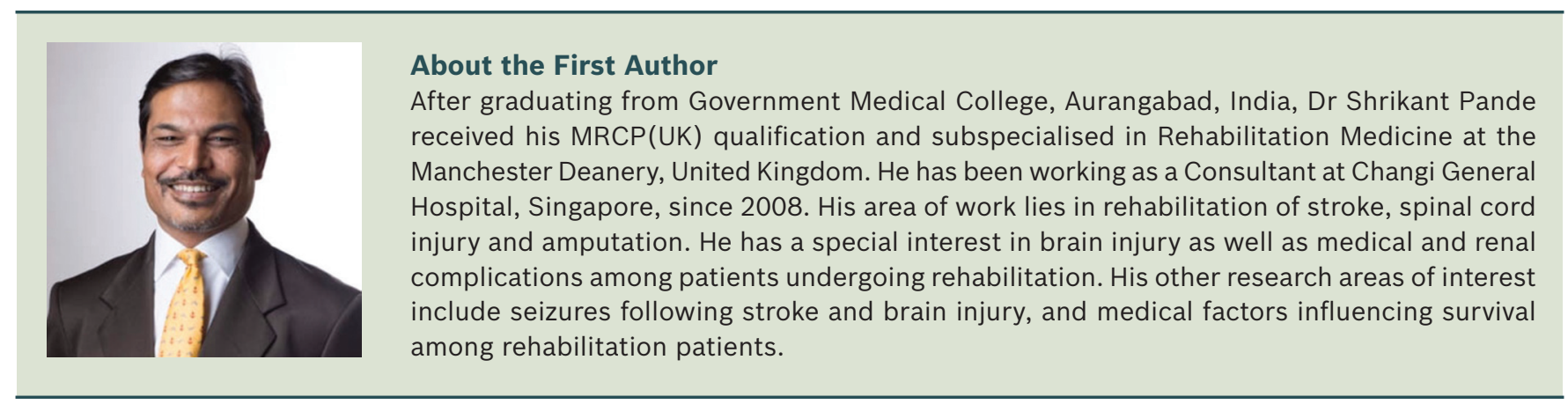

